\title{
Hereditary Hemorrhagic Telangiectasia: Breakpoint Characterization of a Novel Large Deletion in ACVRL1 Suggests the Causing Mechanism
}

\author{
Laura Boeri $^{\mathrm{a}}$ Orietta Radi $^{\mathrm{a}}$ Cecilia Canzonieri ${ }^{\mathrm{a}} \quad$ Elisabetta Buscarini ${ }^{\mathrm{d}}$ \\ Agnese Scatigno $^{a} \quad$ Antonella Minellia $^{a}$ Federica Ornati ${ }^{a}$ b Fabio Pagella ${ }^{b}$ \\ Cesare Danesino $^{\mathrm{a}, \mathrm{c}}$ Carla Olivieria \\ a Department of Molecular Medicine, University of Pavia, and ${ }^{b}$ ENT Unit and ${ }^{\mathrm{C}}$ Genetic counseling, IRCCS Policlinico \\ San Matteo, Pavia, and ${ }^{\mathrm{d}}$ Endoscopy Unit, Ospedale Maggiore, Crema, Italy
}

\section{Key Words}

ACVRL1 - Large deletion - MLPA - Short direct repeats .

Slippage

\begin{abstract}
Hereditary hemorrhagic telangiectasia (HHT) is an autosomal dominant vascular dysplasia. Mutations in either ENG or ACVRL 1 account for around $85 \%$ of cases, and $10 \%$ are large deletions and duplications. Here we present a large novel deletion in ACVRL1 gene and its molecular characterization in a 3 generation Italian family. We employed short tandem repeats (STRs) analysis, direct sequencing, multiplex ligation-dependant probe amplification (MLPA) analysis, and 'deletion-specific' PCR methods. STRs Analysis at ENG and ACVRL1 loci suggested a positive linkage for ACVRL1. Direct sequencing of this gene did not identify any mutations, while MLPA identified a large deletion. These results were confirmed and exactly characterized with a 'deletion-specific' PCR: the deletion size is 4,594 bp and breakpoints in exon 3 and intron 8 show the presence of short direct repeats of 7 bp [GCCCCAC]. We hypothesize, as causative molecular mechanism, the replication slippage model. Understanding the fine mechanisms associated with genomic rearrange-
\end{abstract}

ments may indicate the nonrandomness of these events, highlighting hot spots regions. The complete concordance among MLPA, STRs analysis and 'deletion-specific PCR' supports the usefulness of MLPA in HHT molecular analysis.

Copyright $\odot 2013$ S. Karger AG, Basel

Hereditary hemorrhagic telangectasia (HHT) or Rendu-Osler-Weber disease is an autosomal dominant vascular dysplasia that affects 1 in 5-8,000 [Lesca et al., 2007; Govani and Shovlin, 2009; Shovlin, 2010; Faughnan et al., 2011]. A clinical diagnosis of HHT is based on the presence of at least 3 of the following 'Curaçao criteria': (1) spontaneous, recurrent epistaxis; (2) multiple telangiectases at characteristic sites as nose, lips, oral cavity, and fingers tips; (3) visceral lesions, such as arteriovenous malformations in lungs, liver, brain, spinal cord, and GI tract; (4) family history of a first-degree relative with HHT who has been diagnosed with the same criteria [Shovlin et al., 2000]. HHT presentation patterns are highly variable even within families [Govani and Shovlin, 2009]. HHT patients carry mutations in ENG (OMIM 131195) (HHT type 1: OMIM 187300), ACVRL1 (OMIM 601284) (HHT type 2: OMIM 600376) or SMAD4 (OMIM

\section{KARGER}

E-Mail karger@karger.com

www.karger.com/msy (c) 2013 S. Karger AG, Basel

$1661-8769 / 13 / 0043-0119 \$ 38.00 / 0$ 


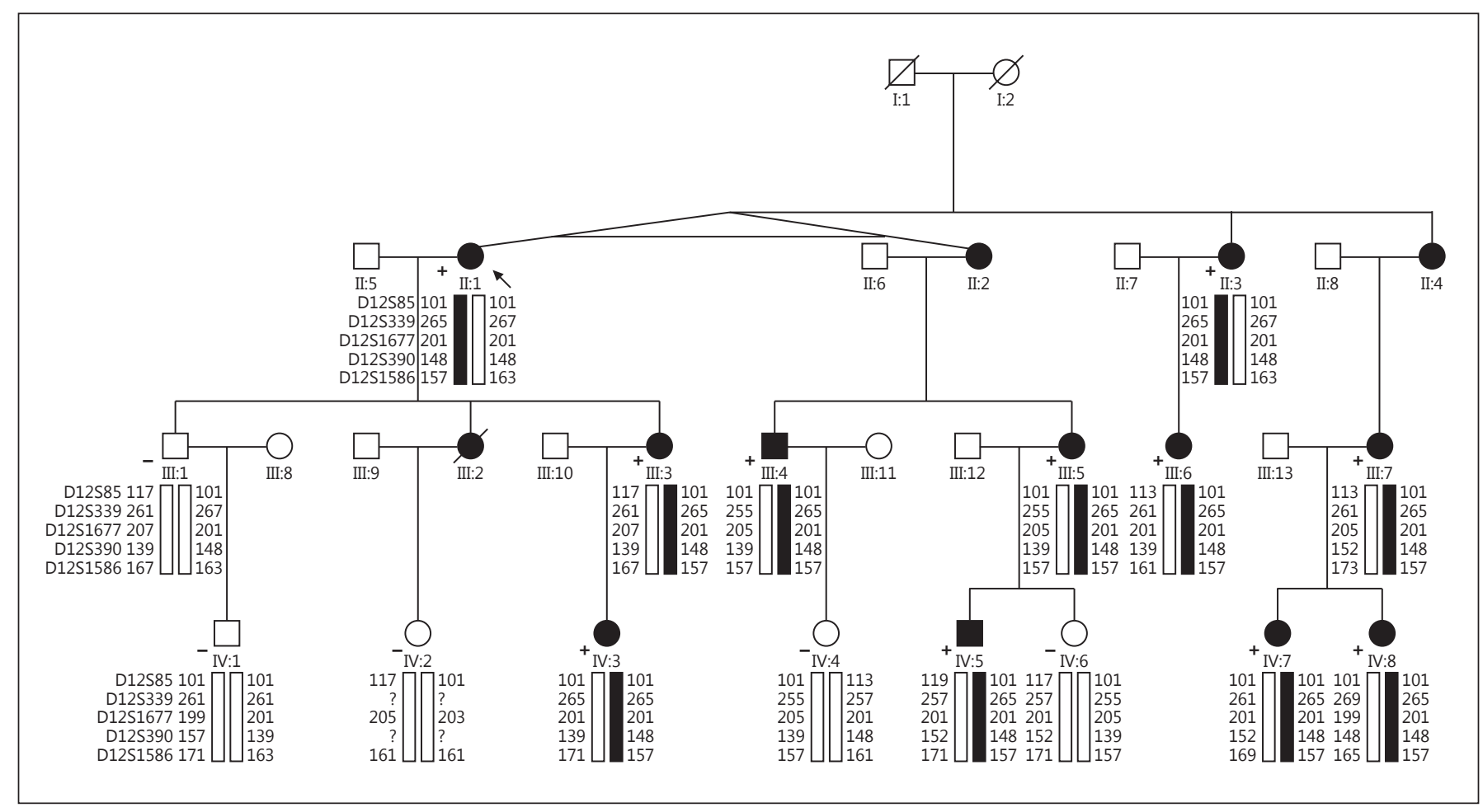

Fig. 1. The pedigree of the family. The proband (II:1) and all analysed affected family members (black-shaded) were positive for the ACVRL1 exon 3-8 deletion. MLPA results are indicated for each family member as $(+)$ or (-) for the presence or absence of the ACVRL1 deletion, respectively. D12S1677 is an intragenic marker located in IVS9.

600973) (HHT in association with juvenile polyposis (JPHT): OMIM 175050) genes [McAllister et al., 1994; Johnson et al., 1996; Gallione et al., 2004].

All these genes belong to the TGF $\beta / B M P s$ pathway [David et al., 2007]. Two additional HHT loci have been reported, but no new disease causing gene has been identified till now [Cole et al., 2005; Bayrak-Toydemir et al., 2006]. Screening for ENG and ACVRL1 mutations by full coding region sequencing and analysis for copy number changes by multiplex ligation-dependent probe amplification (MLPA) or quantitative multiplex-polymerase chain reaction (QM-PCR) identifies mutations for approximately $80-90 \%$ of clinically diagnosed patients [McDonald et al., 2011]. Until now, over 350 mutations in ACVRL1 and 450 mutations in ENG have been described in the HHT mutation database (http://arup.utah.edu/database/HHT/). These mutations, most of which are private, include missense, nonsense, splice site mutations, small deletions or duplications, and insertions [Lesca et al., 2008]. Approximately 10\% of HHT patients have large deletions or duplications [Lesca et al., 2006; Prigoda et al.,
2006; Richards-Yutz et al., 2010; McDonald et al., 2011]. In a few cases, the breakpoints of the rearrangements and the mechanism of origin of the abnormality have been studied [Prigoda et al., 2006; Shoukier et al., 2008; Wooderchak et al., 2010]. These kind of mutations are generally missed by standard PCR amplification and direct sequencing. Here, we report on a novel large deletion in ACVRL1 gene and its molecular characterization in a large Italian family with clinically confirmed HHT.

\section{Materials and Methods}

Blood samples from 16 members (11 affected) of a large HHT Italian family diagnosed according to Curaçao criteria were collected by E.B. A written informed consent was obtained from all subjects. Genomic DNA was extracted from peripheral EDTA-anticoagulated blood samples using standard procedures. Linkage analysis for the ENG locus on chromosome 9 (about $15 \mathrm{cM}$ ) and ACVRL1 locus on chromosome 12 (about $10 \mathrm{cM}$ ) was performed using 6 and 5 short tandem repeat (STR) markers, respectively (STRs ENG: available on request; STRs ACVRL1: see fig.1). Primer 
sequences were found in NCBI UniSTS database (http://www. ncbi.nlm.nih.gov/sites/entrez?db=unists) and forward primers were conjugated with Hex or 6-FAM fluorochrome. Results were analyzed and visualized with Peak Scanner (Applied Biosystems, Foster City, Calif., USA). All coding exons and exon-intron boundaries of ACVLR1 gene (Ref Seq: NG_009549.1) were amplified according to Olivieri et al. [2007] and sequenced. The PCR products were sequenced with ABI PRISM 3730XL Genetic Analyser with Applera BigDye v3.1 sequencing kit (Applied Biosystems). We used the P093-B1 SALSA MLPA kit HHT/PPH1 (MRC-Holland, Amsterdam, The Netherlands) to analyze large deletions or duplications in ACVRL1, ENG and BMPR2 genes according to the manufacturer's instructions. Probe amplification products were analyzed on an ABI PRISM 3100 Genetic Analyser using Liz500 size standard and G5 filter; MLPA peak plots were visualized with Peak Scanner (Applied Biosystems). Normalization and dosage ratios were calculated using Coffalyser (MRC-Holland). Limits of dosage ratios for deletions and duplications are $\leq 0.7$ and $\geq 1.35$, respectively. We designed primer pairs for deletion-specific PCR using free available softwares: Primer3 (http://frodo.wi.mit.edu/primer3/) and Primer-BLAST (http://www.ncbi.nlm.nih.gov/tools/ primer-blast/). To resolve the $5^{\prime}$ and $3^{\prime}$ breakpoints, we performed a long-range PCR (denaturation at $94^{\circ} \mathrm{C}$ for $5 \mathrm{~min}$, denaturation at $98^{\circ} \mathrm{C}$ for $1 \mathrm{~min}$, annealing at $60^{\circ} \mathrm{C}$ for $1 \mathrm{~min}$, elongation at $72^{\circ} \mathrm{C}$ for $6 \mathrm{~min}$ with the latter 3 steps repeated for 35 cycles and an additional elongation step at $72^{\circ} \mathrm{C}$ for $5 \mathrm{~min}$ ) with primers in intron 2 and intron 8 using TaKaRa LA Taq (Takara Bio Inc., Japan) according to manufacturer's instructions. We screened for interspersed repeats in the ACVRL1 locus and the region of the deletion using the software RepeatMasker (http://www.repeatmasker.org/).

\section{Results}

In our HHT family, linkage analysis identified a common haplotype for STR markers around the ACVRL1 gene shared by all affected members (fig. 1), but sequencing of the gene coding region failed to identify any disease-causing mutations. Instead, MLPA identified a large deletion, spanning exons 3-8 of $A C V R L 1$, in the index case (fig. 2a) and familial cosegregation of deletion and disease was then confirmed (fig. 1). With a 'deletion-specific PCR', we found a 4,594-bp deletion; deleted region coordinates on build Hg18 (NCBI 36) are: chr12:g.50593334-50597927 (NG_009549.1:g.10866_15459del) (fig. 2b). 5' breakpoint is located in exon 3,19 bp upstream the MLPA exon 3 probe, and 3 ' breakpoint in intron 8 . We obtained a fine definition of the sequences bordering the breakpoints using direct sequencing. This deletion can be defined as a 'submicroscopic rearrangement' or 'small genomic change' as its size is $<5 \mathrm{Mb}$ [Stankiewicz and Lupski, 2010]. We searched the deletion we found in the Database of Genomic Variants (DGV) (http://www.projects.tcag.ca/variation/), the HHT Mutation Database and the Human Gene Mutation Database (HGMD) (http://www.hgmd.org/).
Two deletions involving exons 3-8 of ACVRL1 gene are present [Prigoda et al., 2006; Richards-Yutz et al., 2010]: the first one [Prigoda et al., 2006] was reported in 2 apparently unrelated families and breakpoint characterization demonstrated different positions both for $5^{\prime}$ end (which fell in IVS2) and 3' end. Breakpoints of the second deletion [Richards-Yutz et al., 2010] were not characterized at a molecular level.

The analysis with RepeatMasker of the ACVRL1 locus revealed a short interspersed element (SINE) density of $22.30 \%$ (Alu density of $8.82 \%$ and mammalian interspersed repeat (MIR) density of $13.47 \%$ ) and a total interspersed repeats density (SINE + LINE) of $24.74 \%$. Within the region of our deletion, we found only SINEs with a density of $24.29 \%$ (Alu density of $12.89 \%$ and MIR density of $11.41 \%)$. The $\mathrm{C}+\mathrm{G}$ content of ACVRL1 locus is $54.28 \%$ and in the deleted region $56.51 \%$.

\section{Discussion}

Standard molecular genetic testing of HHT includes sequencing of the coding regions and analysis for copy number changes for ENG and ACVRL1 genes [Lesca et al., 2006; Prigoda et al., 2006; McDonald et al., 2011]. In our family, STRs analysis at ENG and ACVRL1 loci showed a common haplotype for the ACVRL1 gene in all affected members. Performing MLPA was necessary because the sequence analysis in this gene did not identify any mutations. We found a large deletion that we decided to characterize using a 'deletion-specific PCR'. The 4,594 bp deletion we found results in loss of the intervening sequence between 2 perfect direct 7-bp repeats [GCCCCAC] and elimination of 1 of these repeats (see fig.2). This sequence includes the 'immunoglobulin heavy chain class switch repeat', a short direct repeat of $5 \mathrm{bp}$ [CCCCA] [Chen et al., 2005a, b], which has been reported to mediate slippage mispairing and to cause deletions and translocations [Demura et al., 2002; Abeysinghe et al. 2003]. It is well documented that the slippage mechanism during replication process is mediated by the presence of direct repeats (2-8 bp) at the end points of deletions/duplications and that only one short repeat is retained in the rearrangement [Ketterling et al., 1994; Chen et al., 2005a, b; Taulan et al., 2009]. Several characterized deletions showed locus variability both in the sequence and length of the direct repeats. Probably other factors, such as chromatin higher-order structure [Woodcock and Ghosh, 2010] and chromosomal location of the loci may play an important role in generating these rearrangements [Tau- 

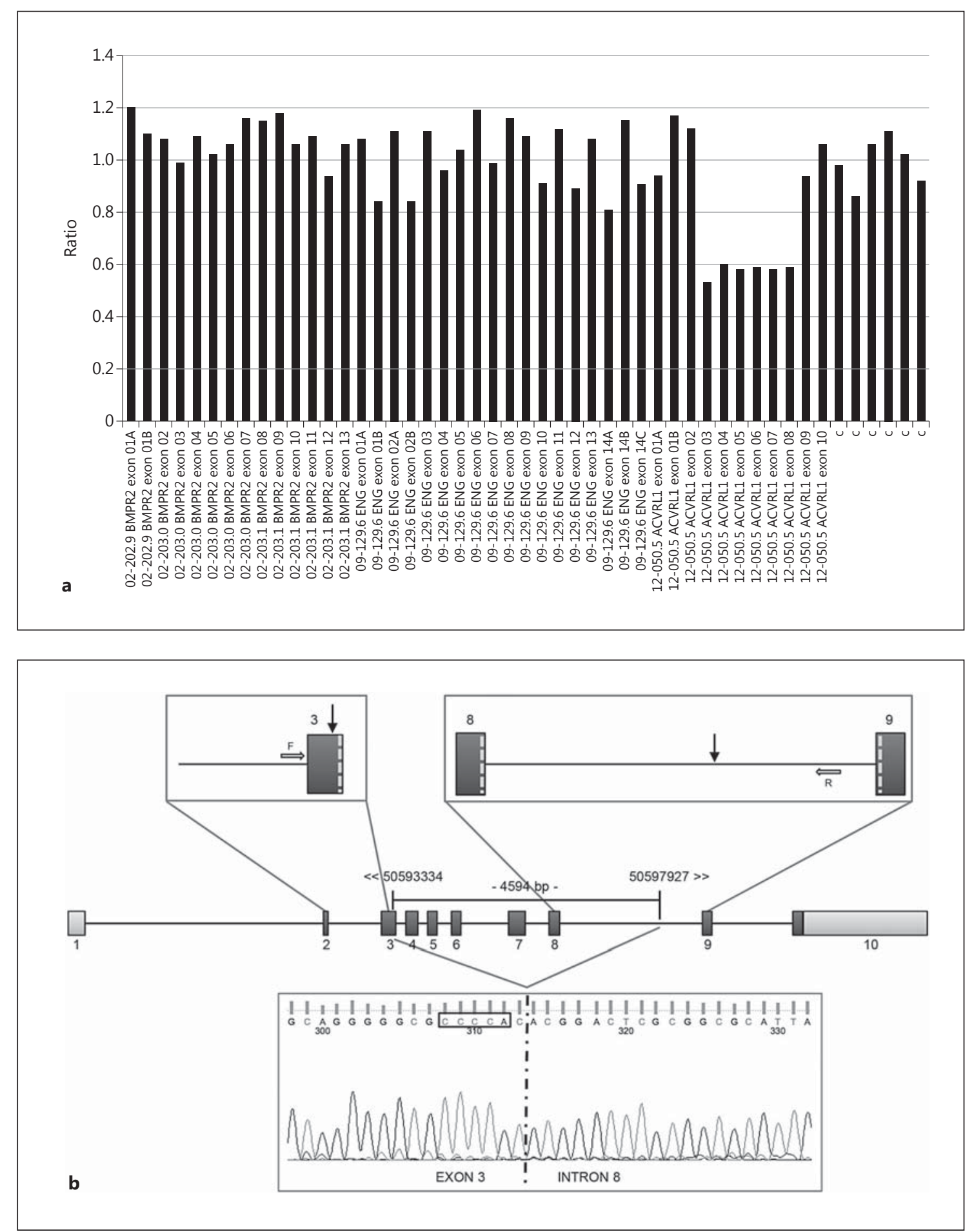

Fig. 2. Characterization of the deletion. a MLPA results obtained with Coffalyser. The ACVRL1 deleted exons 3-8 have a peak ratio $<0.7$. b Schematization of the deleted region and sequence of the PCR product encompassing the breakpoints and showing a deletion of 4,594 bp (Hg18 (NCBI 36): chr12:g.50593334-50597927).
Black vertical arrows in the scheme indicate deletion breakpoints. White dashed vertical lines indicate MLPA probes position. Large horizontal arrows indicate position of primers for deletion-specific PCR. In the electropherogram, the 'immunoglobulin heavy chain class switch repeat' sequence is boxed. 
lan et al., 2009]. Interestingly, using RepeatMasker we found $24.74 \%$ of total interspersed repeats for the ACVRL1 gene, which could cause instability of the region [Resta et al., 2010]. Moreover, the deleted region contains 2 MIRc repeats and $5 \mathrm{MIR}$ repeats and this kind of internal motifs might fold into secondary DNA conformations that could have a role in the slippage model [Bacolla et al., 2006]. Lesca et al. [2006] underlined that the density of Alu repeats that can lead to unequal recombination is higher in the ENG gene than in ACVRL1, and it can explain the higher frequencies of large deletions or duplications in ENG. McDonald et al. [2011] also suggested that repetitive elements would predispose recombination and genomic rearrangements in ENG. A detailed definition of large deletions was reported by Wooderchak et al. [2010], who found a dynamic deletion in ENG and Shoukier et al. [2008], who described 1 deletion in ENG and 4 deletions involving $A C V R L 1$. They suggest non-homologous end joining as the probable mechanism of origin of these deletions because there was little homology between the breakpoint flanking sequences. In this work, we characterized the deletion breakpoints in the ACVRL1 gene and also hypothesized the slippage mispairing as the probable causing mechanism. Understanding the fine mechanisms associated with genomic rearrangements may indicate the nonrandomness of these events, highlighting hot spots regions able to mediate rearrangements [Eyries et al., 2011]. MLPA is a new approach for the detection of genomic rearrangements. This technique has proven to be a useful and reliable diagnostic tool in several genetic disorders [Paracchini et al., 2008; Wooderchak-Donahue et al., 2011]. In HHT molecular analysis, MLPA has been reported to be used on only a small number of samples, thus with limited information about its sensitivity [Shoukier et al., 2008; Wooderchak et al., 2010; McDonald et al., 2011]. For this reason, any rearrangements found with MLPA must be interpreted carefully and verified by alternative techniques, such as 'deletion-specific PCR'.

The complete concordance among MLPA, STRs analysis and 'deletion-specific PCR' for the family tested supports MLPA as an efficient technique. Our results suggest the 'slippage mispairing' as the likely mechanism for the origin of the deletion we studied and emphasize the importance of screening for large rearrangements in HHT patients negative to sequence analysis or in presence of variants with uncertain pathogenicity in $A C V R L 1$ and ENG genes.

\section{Acknowledgement}

We would like to thank the Associazione Fondazione Italiana HHT 'Onilde Carini' for funding L.B., C.C., E.B., C.O., and C.D.

\section{References}

-Abeysinghe SS, Chuzhanova N, Krawczak M, Ball EV, Cooper DN: Translocation and gross deletion breakpoints in human inherited disease and cancer I: nucleotide composition and recombination-associated motifs. Hum Mutat 22:229-244 (2003).

- Bacolla A, Wojciechowska M, Kosmider B, Larson JE, Wells RD: The involvement of non-B DNA structures in gross chromosomal rearrangements. DNA Repair (Amst) 5:11611170 (2006).

- Bayrak-Toydemir P, McDonald J, Akarsu N, Toydemir RM, Calderon F, et al: A fourth locus for hereditary hemorrhagic telangiectasia maps to chromosome 7. Am J Med Genet A 140:2155-2162 (2006).

-Chen JM, Chuzhanova N, Stenson PD, Ferec C, Cooper DN: Complex gene rearrangements caused by serial replication slippage. Hum Mutat 26:125-134 (2005a).

-Chen JM, Chuzhanova N, Stenson PD, Ferec C, Cooper DN: Meta-analysis of gross insertions causing human genetic disease: novel mutational mechanisms and the role of replication slippage. Hum Mutat 25:207-221 (2005b).
Cole SG, Begbie ME, Wallace GM, Shovlin CL: A new locus for hereditary haemorrhagic telangiectasia (HHT3) maps to chromosome 5. J Med Genet 42:577-582 (2005).

David L, Mallet C, Mazerbourg S, Feige JJ, Bailly S: Identification of BMP9 and BMP10 as functional activators of the orphan activin receptor-like kinase 1 (ALK1) in endothelial cells. Blood 109:1953-1961 (2007).

- Demura M, Takeda Y, Yoneda T, Furukawa $\mathrm{K}$, Usukura $\mathrm{M}$, et al: Two novel types of contiguous gene deletion of the AVPR2 and $A R H G A P 4$ genes in unrelated Japanese kindreds with nephrogenic diabetes insipidus. Hum Mutat 19:23-29 (2002).

Eyries M, Coulet F, Girerd B, Montani D, Humbert $\mathrm{M}$, Lacombe $\mathrm{P}$, et al: ACVRL1 germinal mosaic with two mutant alleles in hereditary hemorrhagic telangiectasia associated with pulmonary arterial hypertension. Clin Genet 82:173-179 (2012).

Faughnan ME, Palda VA, Garcia-Tsao G, Geisthoff UW, McDonald J, et al: International guidelines for the diagnosis and management of hereditary haemorrhagic telangiectasia. J Med Genet 48:73-87 (2011).
Gallione CJ, Repetto GM, Legius E, Rustgi AK, Schelley SL, et al: A combined syndrome of juvenile polyposis and hereditary haemorrhagic telangiectasia associated with mutations in MADH4 (SMAD4). Lancet 363:852859 (2004).

Govani FS, Shovlin CL: Hereditary haemorrhagic telangiectasia: a clinical and scientific review. Eur J Hum Genet 17:860-871 (2009).

-Johnson DW, Berg JN, Baldwin MA, Gallione CJ, Marondel I, et al: Mutations in the activin receptor-like kinase 1 gene in hereditary haemorrhagic telangiectasia type 2. Nat Genet 13: 189-195 (1996).

Ketterling RP, Vielhaber EL, Lind TJ, Thorland EC, Sommer SS: The rates and patterns of deletions in the human factor IX gene. Am J Hum Genet 54:201-213 (1994).

Lesca G, Burnichon N, Raux G, Tosi M, Pinson S, et al: Distribution of ENG and ACVRL1 (ALK1) mutations in French HHT patients. Hum Mutat 27:598 (2006).
Characterization of a Novel ACVRL1

Large Deletion
Mol Syndromol 2013;4:119-124 DOI: $10.1159 / 000347029$ 
Lesca G, Olivieri C, Burnichon N, Pagella F, Carette MF, et al: Genotype-phenotype correlations in hereditary hemorrhagic telangiectasia: data from the French-Italian HHT network. Genet Med 9:14-22 (2007).

- Lesca G, Genin E, Blachier C, Olivieri C, Coulet F, et al: Hereditary hemorrhagic telangiectasia: evidence for regional founder effects of ACVRL1 mutations in French and Italian patients. Eur J Hum Genet 16:742-749 (2008).

McAllister KA, Grogg KM, Johnson DW, Gallione CJ, Baldwin MA, et al: Endoglin, a TGFbeta binding protein of endothelial cells, is the gene for hereditary haemorrhagic telangiectasia type 1. Nat Genet 8:345-351 (1994).

-McDonald J, Damjanovich K, Millson A, Wooderchak W, Chibuk JM, et al: Molecular diagnosis in hereditary hemorrhagic telangiectasia: findings in a series tested simultaneously by sequencing and deletion/duplication analysis. Clin Genet 79:335-344 (2011).

- Olivieri C, Pagella F, Semino L, Lanzarini L, Valacca C, et al: Analysis of ENG and ACVRL1 genes in 137 HHT Italian families identifies 76 different mutations (24 novel). Comparison with other European studies. J Hum Genet 52:820-829 (2007)
Paracchini V, Seia M, Coviello D, Porcaro L, Costantino L, et al: Molecular and clinical features associated with CFTR gene rearrangements in Italian population: identification of a new duplication and recurrent deletions. Clin Genet 73:346-352 (2008).

Prigoda NL, Savas S, Abdalla SA, Piovesan B, Rushlow D, et al: Hereditary haemorrhagic telangiectasia: mutation detection, test sensitivity and novel mutations. J Med Genet 43: 722-728 (2006).

Resta N, Giorda R, Bagnulo R, Beri S, Della Mina $\mathrm{E}$, et al: Breakpoint determination of 15 large deletions in Peutz-Jeghers subjects. Hum Genet 128:373-382 (2010).

Richards-Yutz J, Grant K, Chao EC, Walther SE, Ganguly A: Update on molecular diagnosis of hereditary hemorrhagic telangiectasia. Hum Genet 128:61-77 (2010).

Shoukier M, Teske U, Weise A, Engel W, Argyriou L: Characterization of five novel large deletions causing hereditary haemorrhagic telangiectasia. Clin Genet 73:320-330 (2008).

Shovlin CL: Hereditary haemorrhagic telangiectasia: pathophysiology, diagnosis and treatment. Blood Rev 24:203-219 (2010).
-Shovlin CL, Guttmacher AE, Buscarini E, Faughnan ME, Hyland RH, et al: Diagnostic criteria for hereditary hemorrhagic telangiectasia (Rendu-Osler-Weber syndrome). Am J Med Genet 91:66-67 (2000).

Stankiewicz P, Lupski JR: Structural variation in the human genome and its role in disease. Annu Rev Med 61:437-455 (2010).

- Taulan M, Guittard C, Theze C, Claustres M, Georges M: A novel double deletion underscores the importance of characterizing end points of the CFTR large rearrangements. Eur J Hum Genet 17:1683-1687 (2009).

Woodcock CL, Ghosh RP: Chromatin higher-order structure and dynamics. Cold Spring Harb Perspect Biol 2:a000596 (2010).

-Wooderchak-Donahue W, Vaughn C, Chou LS, Lewis T, Sumner K, et al: Verification of multiplex ligation-dependent probe amplification probes in the absence of positive samples. Genet Test Mol Biomarkers 15:793-799 (2011).

Wooderchak W, Gedge F, McDonald M, Krautscheid P, Wang X, et al: Hereditary hemorrhagic telangiectasia: two distinct $E N G$ deletions in one family. Clin Genet 78:484-489 (2010). 